\title{
Colombia Como un "Poder Medio Oceánico": Análisis de las Interacciones del País Dentro de la Región de la Cuenca del Caribe
}

\author{
Felipe Urrego González ${ }^{1}$ \\ Nicolás Correa Ramos ${ }^{2}$ \\ Cristhian Mendoza Pérez ${ }^{3}$
}

\section{Resumen}

En los últimos años, Colombia ha crecido como protagonista en las realidades de América Latina. El país se encuentra en una posición geográfica favorable al encontrarse en la esquina norte de América del Sur, lo que le permite interactuar no solo regionalmente con Sudamérica sino también con Centro América y el Caribe. Además, el reciente acuerdo de paz con un grupo insurgente y el vacío de poder que dejó su desmovilización han desarrollado oportunidades regionales y amenazas no solo a nivel nacional, sino también se debe considerar el impacto de organizaciones delictivas transnacionales a nivel regional. Teniendo en cuenta un enfoque constructivista de seguridad internacional y siguiendo los conceptos de "Complejo de Seguridad Regional" (CSR) y "Poder Medio Oceánico", el presente manuscrito realiza un análisis acerca de cómo el gobierno colombiano ha venido interactuando con otros estados en la Región de la Cuenca del Caribe para generar condiciones de seguridad y defensa común.

\section{Palabras Claves}

Colombia, Análisis, Poder Medio Oceánico, Complejo de Seguridad Regional, Seguridad, Defensa.

\begin{abstract}
In recent years, Colombia has grown as a protagonist in the realities of Latin America. The country is in a favorable geographical position because it is located in the northern corner of South America, which allows it to interact not only regionally with South America but also with Central America and the Caribbean. In addition, the recent peace agreement with an insurgent group and the power vacuum left by its demobilization have developed regional opportunities and threats not only at the national level, but also the impact of transnational criminal organizations at the regional level. Taking into account a constructivist approach to international security and following the concepts of "Regional Security Complex" (CSR) and "Medium Oceanic Power", the present manuscript analyzes how the Colombian government has been interacting with other states in the Caribbean Watersheds Region to generate common security and defense conditions.
\end{abstract}

\section{Keywords}

Colombia, Analysis, Medium Oceanic Power, Regional Security Complex, Security, Defense.

\footnotetext{
1. Estudiante de curso básico, Facultad de Ciencias Navales y Náuticas, Escuela Naval de Cadetes “Almirante Padilla”. Felipe.urrego@armada.mil.co 2. Estudiante de curso básico, Facultad de Ciencias Navales y Náuticas, Escuela Naval de Cadetes "Almirante Padilla". Nicolas.correa@armada.mil.co 3. Coordinador de Investigación, Facultad de Ciencias Navales y Náuticas, Escuela Naval de Cadetes "Almirante Padilla”. Poseidon@enap.edu.co
} 


\section{Introducción}

El interés de Colombia por desarrollar y fortalecer su poder marítimo se ha convertido en una prioridad; prueba de ello es la promulgación de la "Política Nacional de Océanos y Espacios Costeros" (NPOCS) de Colombia en 2017. Esta política apunta a la importancia de desarrollar una estrategia marítima al igual que otras naciones y propone claros ejemplos como los casos de los Estados Unidos, China y la Unión Europea demostrando la importancia de una estrategia marítima para promover el desarrollo económico (Comisión Colombiana del Océano, 2017). Por otro lado, el objetivo del gobierno Colombiano en su estrategia marítima es de convertirse en un "poder medio oceánico" y define este concepto como el nivel de "Smart Power", término que hace alusión a las diferentes herramientas del arte de la gobernanza que involucra el poder blando como la ayuda económica al poder duro como las instituciones militares (Wilson, 2008), que el estado debe poseer para la consecución de su interés, que en este caso es el uso de una explotación de su territorio marino (Comisión Colombiana del Océano, 2017). Dado lo anterior, ha existido un interés especial en mejorar la posición de Colombia a nivel regional en materia de seguridad marítima, es por esto que la presente investigación se centra en el análisis de las relaciones adelantadas por el gobierno colombiano con los países de la región, en aras de organizar e implementar una agenda de trabajo conjunto, continuo y prolongado, en materia de seguridad y defensa internacional, teniendo en cuenta las capacidades actuales de la Armada Nacional.

\section{Metodología}

Para el desarrollo del presente trabajo se implementó un análisis cualitativo de varias fuentes de información que den muestra de las estrategias de seguridad marítima emprendidas por el gobierno colombiano. Esto incluye recursos académicos, políticas gubernamentales, periódicos, artículos de revistas y experiencias personales (Quintana \& Montgomery, 2006). Dicho análisis se centró en la manera de cómo el gobierno colombiano ha "seguritizado" su

Ediciones EFIM dominio marítimo y cómo interactúa con otros estados dentro del marco del CSR en aspectos económicos, militares, ambientales y sociales. En ese orden de ideas, este estudio en primera instancia se presenta una breve introducción al concepto de CSR de Buzan y Weaver (2003) y el concepto de seguridad marítima. En segundo lugar, se analiza cómo Colombia se ha comprometido con otros estados dentro de la CSR en diferentes aspectos que promueven la seguridad, y, por último, con base en el levantamiento de información y su análisis posterior, se plantean algunas recomendaciones sobre cómo mejorar la posición de Colombia dentro del CSR en aspectos de seguridad marítima.

\section{Resultados y Discusión}

\section{“Complejo de Seguridad Regional" y segu- ridad marítima}

Este artículo utiliza un enfoque constructivista del concepto de seguridad internacional para analizar y establecer las relaciones que mejoran las posibilidades de que Colombia sea un actor más activo en la construcción de la seguridad en la región. En ese sentido, el concepto de CSR propuesto por Buzan y Weaver (2003) se utiliza como una forma de comparar cómo el Estado colombiano se involucra en Centroamérica y el Caribe para producir y mejorar sus probabilidades de convertirse en un "poder medio oceánico". Este concepto también ayuda a identificar las conexiones entre los diferentes estados de la región y proporciona una mejor imagen y comprensión de las relaciones y dinámicas de seguridad dentro de la región (Kilroy, Rodriguez Sumano, y Hataley, 2017) (Stone, 2009). De igual forma, es importante llevar el concepto de seguridad marítima ya que este artículo busca usar la teoría CSR en ese contexto. Para tal efecto, se sigue el concepto de Bueger (2014), el cual define la seguridad marítima como una forma de reducir las amenazas en el dominio marítimo y aprovecha los aspectos de la "seguritización" de los océanos para ayudar a definir qué es lo que significa brindar seguridad en este entorno.

La seguridad es un aspecto de especial relevancia en los asuntos internacionales de hoy y además juega un papel importante no solo para 
los Estados sino también para diferentes grupos humanos e individuos. En consecuencia, se puede entender que el concepto de seguridad tiene implicaciones en todos los niveles de la vida humana, tanto de forma individual como en forma colectiva. Según Gallie (1956), el concepto de seguridad es altamente controvertido, esto en el sentido de que tiene diferentes significados según quién proponga su definición y a quienes afecte. Además, dentro del marco de las relaciones internacionales, la seguridad se define como la capacidad de reducir las amenazas a los valores o intereses del estado convirtiéndolo en un concepto altamente político que desarrolla respuestas sobre qué, cómo y quién es la amenaza (Lasswell, 1936; Williams, 2008). Al observar lo que puede significar la seguridad marítima, se puede dar una definición como la mitigación a las amenazas en el dominio marítimo, lo cual plantea los interrogantes sobre ¿De quién debe estar seguro de las amenazas?, ¿Cuáles son las amenazas en el mar?, Y ¿Cómo se reducen esas amenazas?; similar al concepto de seguridad, la seguridad marítima tiene múltiples definiciones dependiendo de quién propone la definición de amenazas y como desarrollar la seguridad a nivel marítimo.

La evolución de cómo se teoriza la seguridad ha tenido una gran influencia en la seguridad marítima lo cual ha llevado a una evolución hasta darnos el concepto actual. La seguridad marítima ha incluido desde el concepto de poder marítimo siendo este la capacidad militar del estado para proteger y utilizar el mar en su propio interés, como, por ejemplo, la necesidad de la protección de las líneas marítimas comerciales para el crecimiento de la economía y ha llegado a ser un concepto más elaborado donde también se consideran aspectos ambientales y sociales de la seguridad. En consecuencia, se define la seguridad marítima como la capacidad de tener poder marítimo (capacidad militar), seguridad marítima (medio ambiente), economía azul (desarrollo económico) y resiliencia (seguridad humana) por el interés del estado (Bueger, 2014).

Por otro lado, el concepto de "Complejo de Seguridad Regional" fue propuesto inicialmente por Buzan y Waever (2003) definiéndolo como
"Un conjunto de unidades cuyos principales procesos de seguritización, Des-seguritización o ambos están tan interrelacionados lo cual hace que sus problemas de seguridad no pueden ser analizados o resueltos de forma individual". Cuando se habla de un conjunto de unidades se refiere a un grupo de estados en una región geográfica e interconectados por problemas que representan una amenaza para su seguridad en los sectores político, económico, ambiental y militar y les permite enfrentar estas amenazas de forma conjunta (Kilroy, Rodriguez Sumano , \& Hataley, 2017; Stone, 2009); Williams, 2008). Estas conexiones regionales ayudan a comprender y analizar en qué capacidad un estado interactúa y "seguritiza" los problemas, lo que da pistas sobre el papel que juega este estado y la cantidad de poder que tiene en la región.

\section{Las interacciones de Colombia dentro del Caribe CSR}

Como se mencionó anteriormente, la ubicación de Colombia lo coloca en un lugar estratégico para influir no solo en América del Sur sino también en América Central y el Caribe. El gobierno colombiano está involucrado en diferentes mecanismos de integración regional para promover el entendimiento mutuo, la cooperación y el desarrollo, estableciendo un terreno común con otros países de la Gran Cuenca del Caribe (Cancillería de Colombia, 2018). En consecuencia, Colombia no solo apunta a una definición única de región como un país latinoamericano, sino que también ve la importancia de ser miembro de diferentes acuerdos regionales. Es por esto que el país participa activamente en el Caribe como miembro de la Asociación de Estados del Caribe (ACS), en el Pacífico en la Alianza del Pacífico, y en América Central como observador del Sistema de Integración Centroamericana (SICA). Además, Colombia es miembro de la Comunidad de Estados Latinoamericanos y del Caribe y de igual forma de la UNASUR. Toda esta gama de asociaciones y sistemas de integración interconecta a Colombia con otros estados, compartiendo inquietudes y soluciones para una variedad de amenazas. 
Colombia Como un "Poder Medio Oceánico": Análisis de las Interacciones del País Dentro de la Región de la Cuenca del Caribe

Con respecto al interés marítimo y su participación en la Región, Colombia tiene costas en el Océano Pacífico y el Mar Caribe, lo que hace de estos dos un interés vital para el desarrollo del país. En consecuencia, el RSC puede considerarse como la región en la que actúa la Asociación de Estados del Caribe (AEC) y el Sistema de Integración Centroamericana (SICA). Ambos organismos crean espacios para el entendimiento común entre los estados miembros. El primero, AEC, es un espacio para acuerdos económicos para el desarrollo mutuo y sostenible; sino también para facilitar e integrar un sistema de asistencia humanitaria en caso de riesgos ambientales (Asociación de Estados del Caribe, 2018). Y el último, Sistema de Integración Centroamericana (SICA), Colombia no participa como miembro permanente sino como observador regional, lo cual le permite interactuar en temas de seguridad y cooperación contra grupos delictivos organizados dentro de la Estrategia de Seguridad de Centroamérica (Cancillería de Colombia, 2018; Sistema de Integración Centroamericana, 2018). Por lo tanto, para analizar el alcance de Colombia como miembro de un CSR en aspectos de seguridad marítima, estos dos sistemas de agrupación ayudan a analizar la dinámica militar, económica, ambiental y social de la Región del Caribe.

La seguridad marítima tiene un papel importante en la definición y evaluación del CSR en el Caribe y América Central. Según este concepto, el dominio marítimo tiene un objetivo principal para todos los estados miembros de la región. En primer lugar, debido a su situación geográfica como países insulares o con el océano como la única forma de mejorar las conexiones comerciales con otras partes del mundo. Segundo, la mayoría de los estados de la región tienen recursos naturales y perspectivas de desarrollo económico directamente relacionados con la protección y explotación de sus zonas económicas exclusivas, puertos y activos marítimos para mejorar la competitividad y la reducción de la pobreza (Jossep, 2018; Caribbean Development Bank, 2016). Además, la seguridad marítima implica no solo seguridad para el desarrollo económico sino también protección del medio ambiente, la seguridad nacional y Ediciones EFIM la seguridad humana que requiere no solo interactuar para crear condiciones de desarrollo económico sino también reducir las amenazas en la región en referencia a estos otros aspectos de seguridad (Bueger, 2014; Dirección General Marítima [DIMAR], 2013; París, 2001).

Colombia se ha convertido en un socio más activo en la región de la Cuenca del Caribe al promover no solo ejercicios de militares para la seguridad mutua sino también la creación de espacios multilaterales y bilaterales para el entendimiento mutuo en otros aspectos. Después del 11 de noviembre y por promoción de los Estados Unidos, Colombia ha participado en la guerra contra el terrorismo. Por lo cual, no solo ha recibido ayuda militar para aumentar la seguridad dentro de sus fronteras sino también apoyo para mejorar las condiciones sociales y reducir la participación en la producción y tráfico de drogas (Reinoso, Ávila, y Culma, 2017). De igual forma, el esfuerzo internacional de Colombia en la región no solo busca una mayor participación en los acuerdos militares, sino también en las alianzas comerciales, ambientales y de desarrollo hacia una mejora más integral de la región del Caribe.

\section{EI aspecto militar}

Teniendo en cuenta el creciente compromiso de los países del hemisferio occidental con la guerra contra el terror después de los ataques del 11 de noviembre, Colombia lidera la integración y el entendimiento común de las fuerzas militares en la cuenca del Caribe para superar diferentes problemas de seguridad. Colombia produjo múltiples acuerdos de cooperación militar con diferentes países de América Central y el Caribe. Cabe destacar que Colombia hizo acuerdos de cooperación marítima, militar y técnica con países como México, Guatemala, El Salvador, Honduras y República Dominicana. Además, como miembro de la AEC y observador en CARICOM, Colombia tiene compromisos de cooperación militar para el beneficio mutuo en ambos sistemas regionales (Reinoso, Ávila, y Culma, 2017; Cancillería de Colombia, 2018; Withers, 2010). Estos acuerdos y asistencia militar incluyen el control del tráfico de drogas, 
ejercicios marítimos y apoyo técnico en equipos militares. En consecuencia, los esfuerzos realizados por las naciones centroamericanas en "la guerra contra las drogas" han aumentado el número de eventos de interdicción y tamaño de las drogas en el Mar Caribe (Bobea, 2015). Por lo tanto, podría acordarse que Colombia tiene un aporte directo no solo como una solución sino también como el principal productor que crea problemas de seguridad en la Cuenca del Caribe y especialmente en el Caribe occidental donde el tráfico de drogas y el crimen organizado inflige una amenaza directa para la seguridad (Cancillería de Colombia, 2018).

\section{El aspecto económico}

En el aspecto económico, la región del Caribe se encuentra en una posición para interactuar e integrar esfuerzos para el crecimiento mutuo. En ese sentido, la política internacional de asuntos económicos de Colombia apunta a la apertura comercial entre otros estados, especialmente en Centroamérica y el Caribe. En particular, Colombia y la CARICOM suscribieron un acuerdo de cooperación mutua en materia de comercio y cooperación técnica, con el objetivo de "acelerar el proceso de integración caribeño y latinoamericano y de la importancia otorgada por las Partes a los diversos procesos de integración subregional, como un medio lograr una mayor competitividad internacional de la Región y facilitar su pleno desarrollo "(Organización de los Estados Americanos, 1994). Además, Colombia creó un acuerdo de libre comercio con Honduras, Guatemala y El Salvador desde 2007 para promover la inversión, el desarrollo y la cooperación mutua entre las partes signatarias (Organización de los Estados Americanos, 2018). Teniendo en cuenta la política exterior de Colombia durante la mayor parte del siglo XX, el país tiene un papel más activo en la creación de un mercado común para el desarrollo de la región. Últimamente, el gobierno colombiano invirtió en infraestructura marítima en ocho puertos diferentes, no solo en el Mar Caribe sino también en el Pacífico Colombiano, lo que permitió un mayor comercio marítimo con el compromiso regional de mejorar la infraestructura marítima (Sistema Económico Latinoame- ricano y del Caribe, 2015; Banco Interamericano de Desarrollo, 2014; Agencia Nacional de Infraestructura, 2018). Como resultado, Colombia se ha vuelto más competitiva y está más conectada con la Región del Caribe, lo que mejora la participación y el crecimiento económico.

\section{El aspecto ambiental}

En los últimos años, la región del Caribe se ha organizado para la protección de sus estados contra amenazas ambientales como lo son tormentas tropicales, huracanes, tsunamis y cualquier otro peligro que represente amenaza a las poblaciones costeras. Colombia lidera o es miembro activo de muchas iniciativas para la protección del medio ambiente y también la protección de las comunidades contra los peligros ambientales. Dentro de su propio territorio marítimo, la iniciativa Sea Flower busca proteger y crear condiciones de sostenibilidad del rico ecosistema biológico que se encuentra en la isla de San Andrés y las diferentes islas cayo que pertenecen a Colombia que no solo es importante para los intereses de Colombia sino también para otros países debido a la ubicación y el impacto que este habitad tiene en la reproducción de peces en el Caribe (Comisión Colombiana del Océano, 2016; Comisión Colombiana del Océano, Asuntos Marinos, 2018). Por otro lado, el gobierno colombiano también busca esfuerzos para mejorar la investigación científica en la Cuenca del Caribe al formar parte del programa "Intercambio Internacional de Datos e Información Oceanográficos" en la iniciativa de la Comisión Oceanográfica Intergubernamental (COI) de la UNESCO que mejora la distribución de información oceanográfica científica para el desarrollo mutuo. De igual forma, dentro de la COI, el Programa de Tsunami tiene una importante contribución colombiana para el estudio y la reducción de los riesgos asociados con los tsunamis en el Caribe (UNESCO, 2018; Comisión Oceanográfica Intergubernamental de la UNESCO, 2018). En definitiva, Colombia ha realizado un esfuerzo importante para la investigación científica y ambiental en la región del Caribe, lo que demuestra la importancia que tiene el medio ambiente marítimo para el desarrollo de la región.

Anfibios ISSN: 2665-1513 


\section{El aspecto social}

El aspecto social también conocido como seguridad humana se refiere a los problemas que amenazan al individuo. En esta dimensión de seguridad, el hambre, el desempleo, el desplazamiento forzado, la salud y la educación pueden considerarse una amenaza sustancial (Bustillo y Velloso, 2016). La seguridad humana es uno de los principales problemas que enfrenta Colombia en la región. En el Caribe y América Central, Colombia participa como observador o socio en CARICOM y CELAC. En ambos, Colombia se ha involucrado en una variedad de temas que mejoran el desarrollo social en el Caribe. Incluso en una escala mayor, durante la Cumbre de las Américas de Cartagena en 2012, Colombia acordó la promoción de políticas hacia un desarrollo social sostenible que incluya la reducción de las desigualdades y la satisfacción de las necesidades básicas (Cumbre de las Américas, 2018). Por otro lado, el problema de la producción de cocaína y su cadena de suministro ayuda a obstaculizar la condición social de las personas aumentando los crímenes y presiones sobre las poblaciones costeras (Oficina de las Naciones Unidas contra la Droga y el Delito, 2017). Otro aspecto de la seguridad humana que afecta no solo a Colombia sino también a Los países del Caribe y América Central son el tema de la trata de personas, la inmigración ilegal y el desplazamiento de refugiados (El Tiempo, 2017; OIM, 2017; ACNUR, 2017). Finalmente, el impacto ambiental debido a los efectos del cambio climático sobre la capacidad de resiliencia de las comunidades y las personas en la Cuenca del Caribe es un factor importante en la seguridad humana. Colombia participó en este tema al contribuir con esfuerzos en la ayuda humanitaria y la investigación científica (Programa de las Naciones Unidas para el Desarrollo, 2016; UNESCO, 2018). Se podría argumentar que Colombia tiene un fuerte compromiso para abordar las amenazas a la seguridad que tienen un impacto directo en las personas y la seguridad en el Caribe (PNUD 2016), pero también es uno de los países que tiene una mayor participación en el aumento de estas amenazas.

Con base en lo anterior, y teniendo en cuenEdiciones EFIM ta la creciente participación de Colombia en el Caribe RSC y el impacto de los factores interestatales en el resto de los estados que conforman la Cuenca del Caribe, el gobierno colombiano debe considerar los siguientes aspectos para tener mayores cambios y convertirse en un "poder medio oceánico":

- Colombia debería aumentar su poder naval y sus activos militares y continuar cooperando y entrenando personal de otros países para promover un entendimiento común y facilitar operaciones militares conjuntas en asuntos regionales. - El gobierno colombiano debería crear un plan estratégico para impulsar la cooperación económica con los estados del Caribe Oriental o estableciendo un acuerdo económico más elaborado con la CARICOM. - Seguir promoviendo la investigación científica en la cuenca del Caribe con la participación de otros estados, para mejorar las alertas tempranas de peligros ambientales y también para promover una explotación sostenible del mar. - Además de fortalecer su posición internacionalmente; Colombia debería trabajar en la estabilidad del país. Considerando el acuerdo de paz con las FARC, Colombia debe intensificar los esfuerzos para reducir la producción de drogas y el tráfico de drogas a otros países. - Por último, el gobierno debería trabajar en la construcción de la igualdad entre las poblaciones costeras para reducir la capacidad de los grupos del crimen organizado para contratarlos y utilizarlos para operaciones ilegales.

\section{Conclusiones}

En conclusión, Colombia incrementó su participación en la Cuenca del Caribe no solo militarmente, sino también considerando los problemas sociales, económicos y ambientales que afectan a la mayoría de los estados en esta región. Teniendo en cuenta la teoría de "Complejo de Seguridad Regional” presentado por Buzan, existe una interacción entre Colombia, los Estados del Caribe y los Estados centroamericanos para cooperar y proporcionar un frente común 
contra las diferentes amenazas a su seguridad marítima. Todos los países de esta región están aumentando su compromiso con una seguridad marítima única que les permita crear condiciones de crecimiento económico y social. $\mathrm{Y}$ es en ese aspecto, Colombia está mostrando más compromiso para mejorar su presencia en el Caribe y cooperar para el beneficio común de la región. En consecuencia, el Estado sudamericano está avanzando para obtener una posición de liderazgo y de "poder medio oceánico".

\section{Referencias bibliográficas}

Agencia Nacional de Infrastructura. (2018, 05 26). Listado de proyectos. Retrieved from http:// www.ani.gov.co/puertos2

Association of Caribbean States. (2018, 05 23). Association of Caribbean States. Retrieved from http://www.acs-aec.org/index.php?q=es/ sobre-la-aec

Bobea, L. (2015). Criminalidad compleja, ecosistemas transgresores violentos y estadotropismo en el Caribe. In C. N. Guarnizo, Anuario 2015 de la seguridad regional en América Latina y el Caribe (pp. 65-91). Bogotá: Friedrich-Ebert-Stiftung en Colombia.

Bueger, C. (2014). What is maritime security? Marine Policy, 53, págs. 159-164.

Bustillo, I., \& Velloso, H. (2016). Insecurity and development in Latin America and the Caribbean. PRISM, 48-67.

Buzan, B., \& Waever, O. (2003). Regions and powers: the structure of international security. London: Cambridge University Press.

Cancilleria de Colombia. (2018, 05 30). La comunidad del Caribe. Retrieved from http:// www.cancilleria.gov.co/international/consensus/caricom

Cancilleria de Colombia. (2018, 05 14). Politica Exterior. Retrieved from http://www.cancilleria.gov.co/international
Cancilleria de Colombia. (2018, 05 15). Sistema de la Integración Centroamericana. Retrieved from http://www.cancilleria.gov.co/internation$\mathrm{al} /$ consensus/sica

Caribbean Development Bank. (2016). Transforming the caribbean port services industry:towards the efficiency frontier. St Michael: Carribean Development Bank.

Comisión Colombiana del Océano. (2016). Hacia una Potencia Océanica. Bogotá: Comisión Colombiana del Océano.

Comisión Colombiana del Océano. (2018, 5 23). Asuntos Marinos. Retrieved from Reserva de Biósfera Seaflower: http://www.cco.gov.co/ seaflower.html

Comission Colombian Oceanic. (2017). Politica nacional del oceano y los espacios costeros. Bogotá.

Direccion General Maritima (DIMAR). (2013, 07 31). Seguridad Maritima Integral. Retrieved from https://www.dimar.mil.co/content/la-direccion-general-maritima-lidera-fortalecimiento-de-la-seguridad-maritima-integral

El Tiempo. (2017). Migración ilegal se disparó en Colombia en el 2016: 34.000 personas. El Tiempo.

Gallie, W. (1956). Essentially contested concepts. Proceedings of Aristotelian Society, 56, págs. 167-198.

Inter-American Development Bank. (2014). Caribbean regional action plan on freight logistics, maritime transport and trade facilitation. Inter-American Development Bank.

Intergovernmental Oceanographic Commission of UNESCO. (2018). International Oceanographic Data and Information Exchange. Retrieved from https://www.iode.org/

IOM. (2017). Migration and Migrants: Regional Dimensions and Developments. In IOM, World Migration Report 2018. Geneva: IOM.

Anfibios ISSN: 2665-1513 
Colombia Como un "Poder Medio Oceánico":

Análisis de las Interacciones del País Dentro de la Región de la Cuenca del Caribe

Jossep, D. (2018). The Caribbean Council. Recuperado el 1 de 6 de 2018, de Oil, the environment and the Caribbean: https://www.caribbean-council.org/oil-environment-caribbean/

Kilroy, R. J., Rodriguez Sumano , A., \& Hataley, T. (2017). Security inequalities in North America: reassessing regional security complex theory. Journal of Strategic Security, 10(4), págs. 1-28.

Lasswell, H. D. (1936). Politics: Who Get What, When and How. New York: McGraw-Hill.

Organisation of American States. (1994). Sistema de Información sobre Comercio Exterior. Retrieved 05 25, 2018, from Agreement on Trade, Economic and Technical Cooperation between the Caribbean Community (CARICOM) and the Government of the Republic of Colombia: http://www.sice.oas.org/Trade/ CARICOME_1.ASP

Organization of American States. (2018, 05 23). Foreign Trade Information System. Retrieved from Colombia - Northern Triangle: http:// www.sice.oas.org/TPD/COL_Norte/COL_ norte_e.ASP

Paris, R. (2001). Human security: paradigm shif or hot air? International Security, 26(2), 87-102.

Quintana, A., \& Montgomery, W. (2006). Psicología: Topicos de actualidad. Lima: UNMSM.

Reinoso Gonzalez, A. E., Avila Quintero, A. Y., \& Paloma Culma, K. T. (2017). Cooperación en seguridad militar desde Colombia al Gran Caribe: casos representativos. Cuadernos del $\mathrm{Ca}-$ ribe(24).

Sistema de Integracion Centroamericana. (2018, 05 16). Sistema de Integracion Centroamericana. Retrieved from http://www.iila-sica.it/sica/

Sistema Economico Latinoamericano y del Caribe. (2015). Digital ports in Latin America and the Caribbean: situation and prospects. Punta Cana: Sistema Economico LAtinoamericano y del Caribe.
Stone, M. (2009). Security according to $\mathrm{Bu}-$ zan: a comprehensive security analysis. Groupe d'Etudes et d'Expertise "Sécurité et Technologies" GEEST-2009. Paris.

Summit of the Americas. (2018). Follow-up and implementations: mandates. Recuperado el 14 de 5 de 2018, de Social development: http:// www.summit-americas.org/sisca/sd.html

UNESCO. (2018). Tsunami Programme. Retrieved from Portal del Caribe: http://www. ioc-tsunami.org/index.php?option=com_content\&view $=$ article\&id $=9 \&$ Itemid $=15 \&$ lang $=$ es

UNHCR. (2017). Global Report 2017. UNHCR. United Nations Development Programme. (2016). Informe Regional sobre Desarrollo Humano para América Latina y el Caribe. United Nations.

United Nations Office on Drugs and Crime. (2017). World drug report 2017. Vienna: United Nations.

Williams, P. D. (2008). Security Studies. In P. D. Williams, Security Studies: An Introduction (pp. 1-14). New York: Routledge.

Wilson, E. J. (2008). Hard power, soft Power, smart power. The annals of the American academyof political and social science, 616:1. USA. Retrieved from http://journals.sagepub.com/ doi/abs/10.1177/0002716207312618

Withers, G. (2010). Analisis: realciones del comando sur con la region. In M. Donado, \& P. Tibiletti, Atlas Comparativo de la Defensaen America Latina y el Caribe (p. 79). Buenos Aires: Cooperación Internacional. 